\title{
Low-load resistance exercise during inactivity is associated with greater fibre area and satellite cell expression in older skeletal muscle
}

\author{
Daniel R. Moore ${ }^{1 *}$, Ryan P. Kelly ${ }^{2}$, Michaela C. Devries ${ }^{3}$, Tyler A. Churchward-Venne ${ }^{4}$, Stuart M. Phillips ${ }^{4}$, Gianni Parise ${ }^{4}$ \\ \& Adam P. Johnston ${ }^{2}$ \\ ${ }^{1}$ Faculty of Kinesiology and Physical Education, University of Toronto, Toronto, ON, Canada, ${ }^{2}$ Department of Applied Human Sciences, University of Prince Edward Island, \\ Charlottetown, PE, Canada, ${ }^{3}$ Department of Kinesiology, University of Waterloo, Waterloo, ON, Canada, ${ }^{4}$ Department of Kinesiology, McMaster University, Hamilton, ON, \\ Canada
}

\begin{abstract}
Background Age-related sarcopenia is accelerated by physical inactivity. Low-load resistance exercise (LLRE) counters inactivity-induced muscle atrophy in older adults, but changes in muscle fibre morphology are unstudied. We aimed to determine the impact of LLRE during short-term inactivity (step-reduction) on muscle fibre size and capillarity as well as satellite cell (SC) content in older skeletal muscle.

Methods Fourteen older ( 71 years) male adults underwent 14 days of step reduction ( $<1500$ steps/day) while performing six sessions of LLRE ( $30 \%$ maximal strength) with one leg (SR + EX) while the contralateral leg served as an untrained control (SR). Seven healthy ambulatory age-matched male adults ( 69 years) served as a comparator group (COM). Muscle biopsies were taken from the vastus lateralis after 14 days, and immunohistochemical analysis was performed to determine muscle fibre cross-sectional area (CSA), myonuclear content, SC content (PAX7 $7^{+}$cells), and total (C:F) and fibre type-specific (C:Fi) capillary-to-fibre ratios.

Results Type I and II fibre CSA was greater in SR + EX compared with SR. Whereas there were no differences across fibre types between SR + EX and CON, type II fibre CSA was significantly lower in SR compared with COM. Type II myonuclear domain was greater in SR + EX compared with COM and SR. Pax $7^{+}$cells associated with type I and II fibres were lower in SR compared with SR + EX. Type II PAX7+ cells were also lower in SR compared with COM with a similar trend for type I fibres. There were trends for a lower C:Fi in SR compared with SR + EX for both fibre types with no differences for each compared with COM.

Conclusions Minimal LLRE during a period of decreased physical activity is associated with greater muscle fibre CSA, SC content, and capillarization. These results support the use of LLRE as an effective countermeasure to inactivity-induced alterations in muscle morphology with age.
\end{abstract}

Keywords Sarcopenia; Muscle stem cell; Exercise; Inactivity; Ageing; Muscle fibres

Received: 8 November 2017; Revised: 7 March 2018; Accepted: 21 March 2018

*Correspondence to: Daniel R. Moore, PhD, Faculty of Kinesiology and Physical Education, University of Toronto, 100 Devonshire PI, Toronto, ON M5S 2W6, Canada. Email: dr.moore@utoronto.ca

\section{Introduction}

The characteristic loss of muscle mass with ageing, sarcopenia, may precede decrements in muscle function ${ }^{1}$ and appears to be accelerated by muscle disuse. ${ }^{2,3}$ It is well established that muscle disuse (e.g. bed rest or casting) induces profound muscle loss in older adults ${ }^{4,5}$ that is difficult to recover ${ }^{6,7}$ and can have significant consequences for older persons. ${ }^{8}$ We have recently demonstrated that even a short period of inactivity, created by a reduction in daily steps, results in reductions in leg lean muscle mass in as little as 2 weeks. ${ }^{9,10}$ Given that older adults have a delayed and/or 
blunted ability to fully recover lost muscle mass and function following disuse-induced muscle atrophy, ${ }^{6,7}$ it is imperative to elucidate effective countermeasures for its prevention. We recently demonstrated that minimal (six sessions in 14 days) low-load (high effort) resistance exercise (LLRE) maintained leg lean mass, muscle function, and a robust feeding-induced rise in muscle protein synthesis in older adults. ${ }^{10}$ We suggest that these results are related to the ability of LLRE to enhance motor unit recruitment (especially of the higher threshold type II fibres) ${ }^{11,12}$ and ultimately highlight the importance of this training paradigm for maintaining muscle mass and function in older adults. Nonetheless, it is unclear whether and to what degree inactivity-induced changes in muscle mass also affect muscle fibre area and whether there may be a fibre-type specific response.

In addition to alterations in protein metabolism that contribute to the loss of skeletal mass with age and disuse, evidence is accumulating that a contraction of the muscle stem cell [i.e. satellite cell (SC)] pool, and or impaired SC function, may contribute to age-related muscle loss. ${ }^{13}$ This SC dysregulation also appears to be fibre-type specific, with SC associated with fast twitch type II fibres being more 'susceptible' to age-related impairments. ${ }^{14,15}$ This is notable given the generally greater atrophy of these fibres during normal ageing. ${ }^{16,17}$ Additionally, SC are also generally located in close proximity to capillaries, ${ }^{18}$ which would ostensibly ideally position them to respond to circulating regulatory factors (e.g. IGF-1, IL-6, and hepatocyte growth factor). However, SC in older muscle are located at a greater distance from capillaries than in young individuals, which may impair their biological function and, ultimately, the plasticity of skeletal muscle with age. ${ }^{19,20}$ Moreover, the age-related decrease in muscle capillarization may impair nutrient delivery to skeletal muscle, ${ }^{21}$ which could be a contributing factor to both an impairment in muscle metabolism and SC function. ${ }^{19,22,23}$

The main aim of the present study was to investigate the impact of minimal LLRE during 2 weeks of inactivity on skeletal muscle fibre morphology in older adults. We also compared the changes in muscle fibre area, SC content, and capillarization with healthy, ambulatory controls to further elucidate the beneficial effects of LLRE during a period of inactivity in ageing. Our main thesis was, consistent with our previous findings at the whole muscle level, ${ }^{9,10}$ that LLRE would prove to be effective in preserving muscle fibre area with inactivity.

\section{Methods}

\section{Participants}

We examined a subset of 14 healthy, moderately active (7011 \pm 830 steps/day) older men (71 \pm 5 years,
$84.3 \pm 17.3 \mathrm{~kg}, 25.3 \pm 6.1 \%$ body fat) who were recruited to participate in the step-reduction portion of the study, as described previously. ${ }^{10}$ A subset of seven older men from separate study ${ }^{24}$ served as healthy, ambulatory (5649 \pm 2465 steps/day) comparators (COM; $69 \pm 3$ years, $76.6 \pm 7.1 \mathrm{~kg}$, $22.9 \pm 4.5 \%$ body fat). All baseline characteristics were similar between groups (all $P \geq 0.22$; unpaired $t$-test). Participants provided informed consent with the study conforming to the Declaration of Helsinki and also being approved by the Hamilton Integrated Research Ethics Board.

\section{Study design}

Participants were required to reduce their daily steps to $<1500 /$ day for 14 days while performing six sessions of unilateral (i.e. single leg) low-load, high effort (i.e. to volitional fatigue or failure to maintain required range of motion) resistance exercise (i.e. three sessions/week with at least $48 \mathrm{~h}$ between sessions) with a randomly selected leg, as previously described. ${ }^{10}$ This model resulted in a within-participant comparison to determine how exercise during step reduction (SR + EX) influences muscle fibre characteristics and SC content compared with a leg that did not perform exercise (SR). The low-load (30\% of maximal strength) and low-volume (three sets each of leg press and knee extension) exercise was performed to volitional fatigue to minimize the potential for musculoskeletal injuries in this older population yet maximize muscle fibre recruitment, especially of the type II muscle fibres, and muscle protein synthesis. ${ }^{11,25}$ On average, participants completed $\sim 30$ repetitions per set for knee extension and $\sim 35$ repetitions per set for leg press. Muscle biopsies were taken from the vastus lateralis of both legs immediately after the 14-day step-reduction, which occurred $72 \mathrm{~h}$ after the final exercise, bout to determine muscle fibre characteristics and changes in muscle protein synthesis, as described previously. ${ }^{10}$ In order to characterize potential differences from healthy, ambulatory controls, muscle biopsies were taken at rest in a subset of participants from a previous study. ${ }^{24}$ All samples were freed of visible blood and connective tissue prior to being mounted in optimal cutting temperature medium and frozen in isopentane cooled by liquid nitrogen. Samples were stored at $-80^{\circ} \mathrm{C}$ until analysis.

\section{Analysis}

Muscle cross sections $(7 \mu \mathrm{m})$ were prepared from unfixed OCT embedded samples, air dried for 15-45 min, and stored at $-80^{\circ} \mathrm{C}$. Samples were incubated with the following primary antibodies: anti-Pax7 (1:1), anti-laminin (1:500, Abcam), anti-MHCl (1:5, DSHB), and anti-CD31 (1:25, Abcam) followed by washes with phosphate buffered saline $+0.1 \%$ tween (PBS-T). Primary antibodies where revealed 
through immunoflourescent detection with Alexa Flour 488 donkey anti-rabbit (1:500), Alexa Flour 647 donkey antimouse (1:500), Alexa Flour 488 donkey anti-mouse (1:500), and Alexa Fluor 555 donkey anti-rabbit $(1,1000)$ (all Invitrogen). Nuclei were labelled with DAPI prior to final washes $(3 \times 5 \mathrm{~min})$ in PBS-T and cover slipped with fluorescent mounting media. For immunofluorescence stains that detected Pax7, samples were fixed in 4\% PFA for $10 \mathrm{~min}$ followed by washes with PBS-T and blocking with a solution containing $2 \%$ BSA, $5 \%$ FBS, $0.2 \%$ Triton $X-100,0.1 \%$ sodium azide for $60 \mathrm{~min}$ as described previously. ${ }^{14}$ Immunofluorescence stains that detected CD31 were performed as described previously ${ }^{19}$ with slight modifications. Briefly, following air drying, samples were fixed using ice-cold acetone for $10 \mathrm{~min}$ and blocked with 5\% donkey serum. Additionally, a $0.01 \%$ Sudan Black solution (in $70 \% \mathrm{ETOH}$ ) was applied to samples for $10 \mathrm{~min}$ prior to final washes and incubation with DAPI. Samples were visualized with a Zeiss LSM710 laser scanning confocal microscope equipped with a high-resolution camera. For each sample, the following parameters were evaluated as described previously ${ }^{14}$ : muscle fibre cross-sectional area was calculated from at least 75 type-I and type-II fibres, fibre-type distribution was calculated as number of MHC type I fibres/total fibre number, number of myonuclei was identified as fibre-associated, sub-laminin DAPI+ cells while the myonuclear domain was calculated as the number of myonuclei per square micrometre. The total SC number was identified as fibreassociated, sub-laminin, Pax7+ cells while the fibre-type specific quantity of SC was identified as sub-laminin, Pax7+ cells associated with fibres +/- for MHC type I.

Capillaries were quantified from images using FIJ software by the same, blinded individual using areas of the muscle cross-sections spanning $2000 \times 2000$ pixels, which were free of longitudinal fibres and freeze fracture. Analysis consisted of quantification of the capillary-to-fibre ratio in mixed muscle and in a fibre-type specific manner, on both a global (C:F) and local ( $\mathrm{C}: \mathrm{Fi})$ level. These parameters differ as the global measurement gives an indication of the capillarization of the muscle itself, whereas the local parameter reflects the capillarity of the individual fibres. ${ }^{26} \mathrm{C}: \mathrm{F}$ analysis was conducted by counting the total number of capillaries and fibres, along with specific fibre types and their corresponding capillaries. ${ }^{27}$ In order to account for edge effects, fibre counts included all whole fibres within the ROI, while total capillary counts included all interior capillaries plus one-half of all peripheral capillaries. ${ }^{28}$

C:Fi analysis was completed using methods previously described by Hepple et al. ${ }^{29}$ Briefly, 25 fibres of each fibre type were selected within the ROI using the closest 25 fibres of the same type. Capillary contacts (number of capillaries surrounding a fibre) were analysed for each individual fibre, along with each capillary's sharing factor, the number of fibres a capillary comes into contact with.
In order to calculate the $\mathrm{C}: \mathrm{Fi}$, capillaries of a specific sharing factor are multiplied by the reciprocal of their sharing factor and subsequently summed. The formula reads as

$$
\mathrm{C}: \mathrm{Fi}=\left(n_{1}\left(1 / \mathrm{SF}_{1}\right)\right)+\left(n_{2}\left(1 / \mathrm{SF}_{2}\right)\right) \ldots,
$$

where $n$ is equal to the number of capillaries and SF is the corresponding sharing factor.

\section{Statistics}

The primary comparison was determining the difference between SR and SR + EX, which was performed using a twotailed paired $t$-tests with significance accepted at $P \leq 0.05$. To determine differences from COM as a secondary comparison, unpaired $t$-tests were used and adjusted for multiple comparisons (i.e. significance accepted at $P \leq 0.025$ ). A Pearson's product correlation was applied to assess the relationship between SC, muscle protein synthesis, and fibre capillarization with significance accepted at $P<0.05$. Statistics were performed using GraphPad Prism V6.02 software (GraphPad Software Inc., La Jolla, CA, USA). Data presented as means \pm SD.

\section{Results}

\section{Fibre cross-sectional area}

Type I and type II muscle fibre area in SR + EX was $12 \%$ $(P<0.05)$ and $\sim 29 \%$ ( $P<0.01)$ greater, respectively, than in SR (Figure 1A). There was no difference in type I fibre area between COM and SR $(P=0.25)$ and SR + EX $(P=0.74)$. Whereas type II fibre area was $\sim 34 \%$ greater in COM compared with SR ( $P<0.01$; Figure $1 \mathrm{~B})$, there was no difference between $\mathrm{SR}+\mathrm{EX}$ and $\operatorname{COM}(P=0.71$; Figure $1 \mathrm{~B})$.

\section{Myonuclei}

Myonuclei per fibre was similar across all conditions for both type I and type II muscle fibres (all $P \geq 0.16$; Table 1). There was no difference between conditions for myonuclear domain size for type I fibres $(P \geq 0.18)$. In contrast, the similar myonuclei per type II fibre for all conditions resulted in a greater type II myonuclear domain for COM relative to SR $(P<0.01)$ but not relative to $\mathrm{SR}+\mathrm{EX}(P=0.21)$. There was no difference in myonuclear domain between $S R+E X$ and $\operatorname{SR}(P=0.11)$. 
Figure 1 Cross-sectional area of type I (A) and type II (B) muscle fibres during step reduction (SR; $N=14$ ), step reduction with low-load resistance exercise (SR + EX; $N=14)$, and in healthy ambulatory comparators (COM; $N=7)$. Data presented as mean $\left({ }^{\prime}+{ }^{\prime}\right)$, median (line), interquartile range (box), and minimum and maximum (whiskers). ${ }^{*}$ Significantly different, $P<0.05$. ${ }^{*}$ S Significantly different, $P<0.01$.

A.

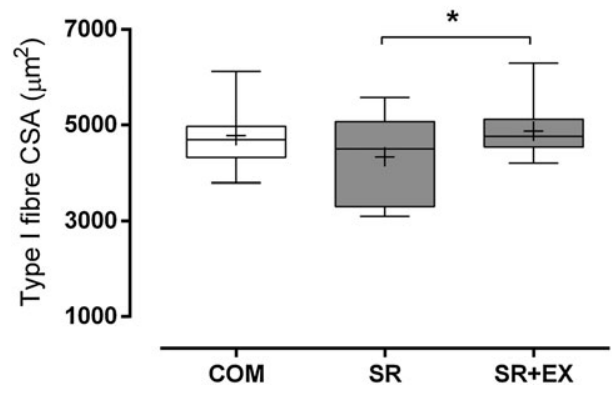

B.

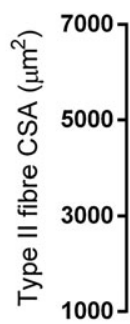

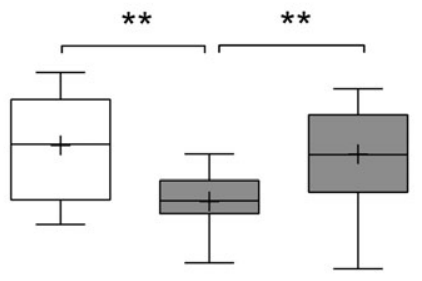

COM SR SR+EX

Table 1 Myonuclei per fibre and myonuclear domain

\begin{tabular}{|c|c|c|c|c|}
\hline & & SR & $\mathrm{SR}+\mathrm{EX}$ & COM \\
\hline Myonuclei per fibre & Type I & $3.0 \pm 0.4$ & $3.1 \pm 0.3$ & $2.9 \pm 0.3$ \\
\hline & Type II & $2.9 \pm 0.5$ & $3.0 \pm 0.4$ & $2.7 \pm 0.2$ \\
\hline Myonuclear domain $\left(\mu \mathrm{m}^{2}\right)$ & $\begin{array}{l}\text { Type I } \\
\text { Type II }\end{array}$ & $\begin{array}{l}1480 \pm 270 \\
1190 \pm 300^{a}\end{array}$ & $\begin{array}{l}1570 \pm 220 \\
1440 \pm 330^{b}\end{array}$ & $\begin{array}{l}1660 \pm 250 \\
1640 \pm 350^{b}\end{array}$ \\
\hline
\end{tabular}

$N=14$ for step reduction (SR) and step reduction with low-load resistance exercise (SR + EX) and $N=7$ for comparator (COM). Conditions with different superscripts are significantly different, $P<0.01$. Means \pm SD.

\section{Satellite cells}

Type I fibre-associated $\mathrm{PAX7}^{+}$cells were lower in SR compared with $\mathrm{SR}+\mathrm{EX}(P<0.01)$ and $\operatorname{COM}(P<0.01$; Figure 2A). There was no difference between $S R+E X$ and COM for type I-associated $\mathrm{PAX7}^{+}$cells $(P=0.39)$. Type II fibreassociated $\mathrm{PAX7}^{+}$cells were lower in SR compared with $\mathrm{SR}+\mathrm{EX}(P<0.01)$ with a trend $(P=0.027)$ towards a difference with $\mathrm{COM}$ (Figure $2 \mathrm{~B}$ ). In contrast, $\mathrm{PAX} 7^{+}$cells were not different between SR + EX and CON $(P=0.26)$.

\section{Capillarization}

A total of $153 \pm 12,139 \pm 11$, and $114 \pm 7$ fibres were analysed for SR, SR + EX, and COM, respectively. Capillary density was similar between conditions $\left(S R=244 \pm 68 \mu \mathrm{m}^{2} /\right.$ capillary; $\mathrm{SR}+\mathrm{EX}=248 \pm 81 \mu \mathrm{m}^{2} /$ capillary; $\mathrm{COM}=234 \pm 36 \mu \mathrm{m}^{2} /$ capillary, $P \geq 0.67)$. Global C:F was greater in SR + EX compared with SR (1.24 \pm 0.19 vs. $1.12 \pm 0.15$, respectively; $P<0.05)$. There was a trend for a difference in global $\mathrm{C}: \mathrm{F}$ between $\operatorname{COM}(1.45 \pm 0.32)$ and $\mathrm{SR}+\mathrm{EX}(P=0.07)$ that reached

Figure 2 Representative images (A) of single channel stains for myosin heavy chain (MHC)1, and Pax7 and merged stain of Pax7/laminin (LAM) and $\mathrm{Pax} 7 / \mathrm{LAM} / \mathrm{MHCl} / \mathrm{DAPI}$ of a muscle cross section. Scale bar $=50 \mu \mathrm{m}$. Quantification of the number of satellite cells (Pax $7^{+}$myonuclei/fibre) in type I (B) and type II (C) muscle fibres during step reduction ( $S R ; N=14$ ), step reduction with low-load resistance exercise $(S R+E X ; N=14)$, and in healthy ambulatory comparators (COM; $N=7)$. Data presented as mean $\left({ }^{\prime}+\right.$ '), median (line), interquartile range (box), and minimum and maximum (whiskers). **Significantly different, $P<0.01 .{ }^{*}$ Trend for a difference, $P=0.027$.
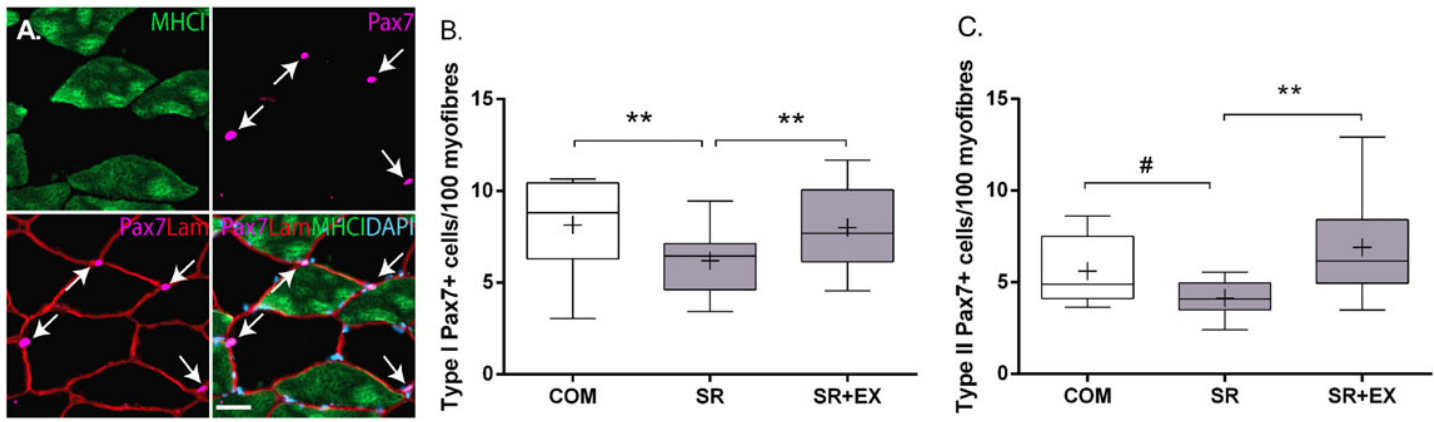
significance with SR $(P<0.01)$. There were trends towards a greater C:Fi between SR + EX compared with SR for type I ( 12\%; $P=0.06)$ and type II ( 13\%; $P=0.09)$ fibres (Figure $3 \mathrm{~A}, 3 \mathrm{~B}$ and $3 \mathrm{C}$ respectively). Sensitivity analysis revealed that the difference between SR and SR + EX for C:Fi became significant $(P<0.01)$ after removal of an outlier, as defined by being greater than 2 SD from the mean fold difference between conditions. There was no difference in C:Fi between COM and SR or SR + EX for type I $(P \geq 0.27)$ or type II $(P \geq 0.14)$ muscle fibres.

\section{Correlations}

There were moderate to large $(r=0.45-0.62$; all $P \leq 0.016)$ correlations between average fibre area, average SC content, and fasted myofibrillar protein synthesis (Figure $4 A-C$ ) when collapsed across SR and SR + EX. There was a moderate $(r=0.41 ; P<0.05)$ correlation between C:Fi and postprandial myofibrillar protein synthesis when collapsed across SR and SR + EX (data not shown). Correlations between C:Fi and SC expression revealed no difference in type I fibres ( $r=0.06 ; P=0.77$ ) but a potential trend in type II fibres $(r=0.32 ; P=0.09)$ in SR and SR + EX.

\section{Discussion}

Muscle disuse results in the loss of lean mass across the lifespan ${ }^{30,31}$ and can have profound health and mobility consequences particularly in older persons. ${ }^{8}$ We previously demonstrated that a reduction in daily steps, as a model of inactivity and partial muscle disuse, leads to the development of anabolic resistance of muscle protein synthesis (i.e. a relative refractoriness of muscle protein synthesis to hyperaminoacidaemia) and the loss of skeletal muscle mass in older adults. ${ }^{9,10}$ Our results here extend those of our previous observations by demonstrating that LLRE (with high effort) not only attenuates inactivity-induced muscle loss in older adults ${ }^{10}$ but also is associated with a greater muscle fibre cross-sectional area, particularly in type II fibres. Moreover, type I and II fibre area in the SR + EX leg was similar in size to healthy age-matched fully ambulatory older adults (COM). In contrast, type II fibre area in SR was significantly less than ambulatory controls, demonstrating that LLRE may be an effective countermeasure to mitigate type II muscle fibre atrophy associated with inactivity. Our results are fundamentally aligned with, for example, the ability of neuromuscular electrical stimulation to attenuate muscle and fibre

Figure 3 Representative images (A) of single channel stains for myosin heavy chain (MHC)1, CD31, and laminin (LAM) and a merged stain of MHC1/ CD31/LAM/DAPI of a muscle cross section. Scale bar $=50 \mu \mathrm{m}$. Capillary contacts per fibre (C:Fi) in type I (B) and type II (C) muscle fibres during step reduction (SR; $N=14)$, step reduction with low-load resistance exercise (SR + EX; $N=14)$, and in healthy ambulatory comparators $(C O M ; N=7)$. Data presented as mean $\left({ }^{\prime}+\right.$ '), median (line), interquartile range (box), and minimum and maximum (whiskers). " Trend for a difference, $P=0.06-0.09$.
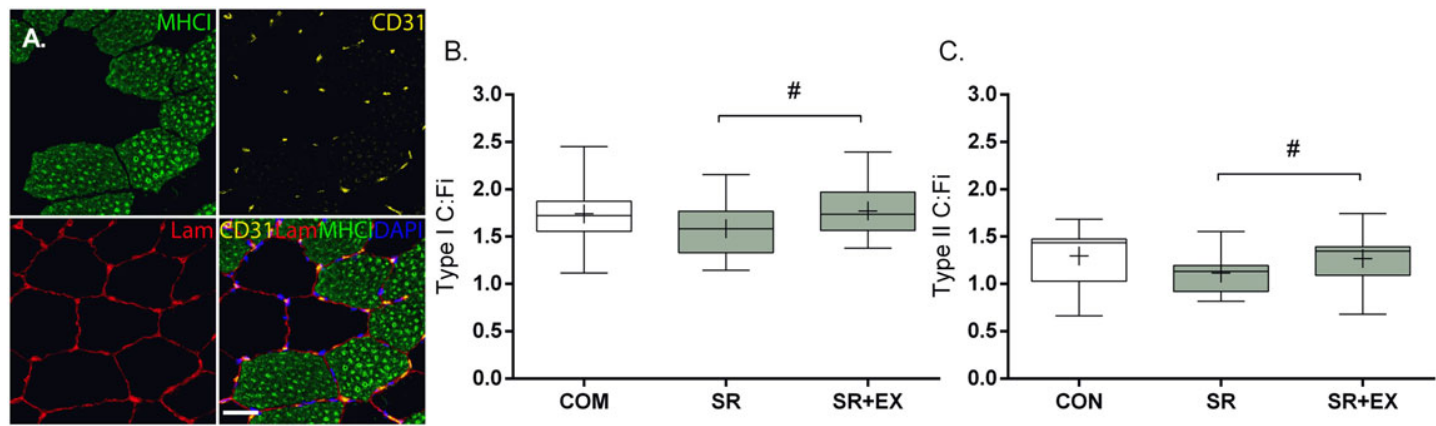

Figure 4 Correlation between average fibre cross-sectional area and fasted myofibrillar protein synthesis rates (A), average PAX7 ${ }^{+}$cells $/ 100$ fibres and average fibre cross-sectional area (B), and average PAX7 $7^{+}$cells $/ 100$ fibres and fasted myofibrillar protein synthesis (C) collapsed across step reduction and step reduction with low-load resistance exercise $\left(N=28\right.$ for all correlations). Fasted myofibrillar protein synthesis determined previously. ${ }^{10}$
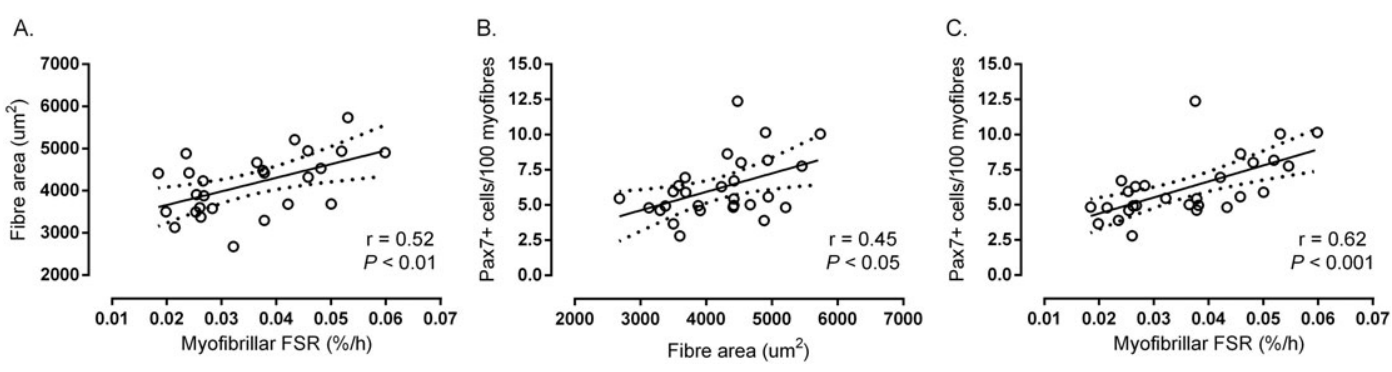
atrophy during bed rest. ${ }^{32}$ The apparent preservation of type II fibre area with unilateral LLRE in the present study is notable given that preferential atrophy of these high forcegenerating and power-generating muscle fibres is a hallmark of age-related muscle loss. ${ }^{16,17}$ Moreover, periods of inactivity have been suggested to accelerate the typical loss of muscle mass with age given that older adults generally experience an attenuated recovery of inactivity-induced muscle loss. $^{6-8}$ Thus, our results and those of others ${ }^{32}$ collectively highlight the importance of some consistent contractile stimulus during even brief periods of inactivity, as with complete disuse, to prevent muscle atrophy in otherwise healthy older adults. As a countermeasure, LLRE may, over time, mitigate or delay the characteristic loss of muscle with age.

Ageing is associated with a general reduction in SC content in skeletal muscle, especially SC associated with type II fibres. ${ }^{33,34}$ Coincident with a lower muscle fibre area, we also observed a greater type I and II fibre SC content in the SR + RE compared with the SR leg after step reduction. Moreover, whereas SC content was similar in SR + EX compared with COM, there was a decreased SC pool size associated with type II fibres in the SR leg. This reduced type II fibre-associated SC content in the SR leg compared with healthy controls suggests that inactivity contributed to a contraction of the SC pool. Whether immobilization results in a contraction of the SC pool in younger adults is debatable ${ }^{35,36}$; however, it appears that older skeletal muscle may be susceptible to inactivity-induced loss of SC. ${ }^{32}$ Mechanistically, the factors responsible for the loss of SC with inactivity are not defined but may be related to cell apoptosis. For example, SC isolated from aged muscle display greater rates of apoptosis concomitant with rises in caspase family members and cell death genes. ${ }^{37}$ In contrast, resistance exercise can induce an expansion of the SC pool in older skeletal muscle despite a delayed type II fibreassociated SC response. ${ }^{14,15}$ Thus, a greater SC content in the present study may be a residual effect of the prior bout of exercise and not necessarily a new basal steady state expansion of the SC pool. Nevertheless, the difference between SC pool sizes and muscle fibre areas in the SR and SR + EX legs is consistent with the observation that SC are decreased in non-exercising older adults, ${ }^{14,15,19}$ which correlates with total muscle mass in this population. ${ }^{38}$ Thus, while our retrospective design precludes our ability to assess whether there was an inactivity-induced contraction of the SC pool and/or an acute/chronic exercise-induced expansion, our data clearly demonstrate that LLRE is associated with a greater SC content associated with both type I and II muscle fibres compared with a leg that is inactive.

The loss of muscle mass with ageing and/or inactivity is underpinned in part by changes in muscle protein metabolism, the most consistent feature of which is an attenuated protein synthetic response in older adults. ${ }^{30,31}$ Given the myofibrillar protein pool represents $\sim 65 \%$ of total skeletal muscle protein, the relationship between fasted myofibrillar protein synthesis and muscle fibre cross-sectional area during inactivity in the SR and SR + RE legs in the present study is perhaps not altogether surprising. ${ }^{30}$ Nonetheless, evidence is accumulating that SC depletion and/or dysregulation is involved in muscle loss during ageing ${ }^{14,38}$ and inactivity. ${ }^{32}$ This notion is consistent with our observation of a relationship between myofibre size and SC content in the present study. Our observation that fasted myofibrillar protein synthesis was correlated with SC content in the SR and SR + RE legs suggest, we propose, these processes may be responding to contractile stimuli in a coordinated manner. ${ }^{39}$ Thus, given that changes in muscle protein synthesis are an important variable influencing muscle mass in otherwise healthy humans, ${ }^{40}$ the relationship between myofibrillar protein synthesis and SC content in the present study is aligned with previous observations that SC content is a modest predictor of muscle mass with ageing. ${ }^{38}$

Muscle tissue microcirculation is important for metabolic health because it can influence the delivery of hormones and nutrients to skeletal muscle. ${ }^{23,41,42}$ We observed a trend ( $P=0.06$ and 0.09 for type I and II fibres, respectively) for a greater capillary contact per muscle fibre in the SR + RE compared with the SR leg during step reduction. Immobilization may decrease ${ }^{43}$ whereas resistance exercise can increase muscle capillarization, ${ }^{29}$ which collectively may function to maintain a constant capillary density. ${ }^{44,45}$ Thus, the difference between the $S R$ and $S R+R E$ legs may be related to the angiogenic nature of the LLRE stimulus in the $S R+R E$ leg and/or an inactivity-induced contraction of the capillary network in the SR leg. The slightly greater capillarization in $\mathrm{SR}+\mathrm{RE}$ compared with SR leg is notable given that the apparent difference is of similar magnitude as the age-induced changes over $\sim 12$ years. ${ }^{21}$ The greater capillary contacts in the $\mathrm{SR}+\mathrm{RE}$ leg may result in greater delivery of circulating nutrients and hormones, which would be consistent with previous observations of a greater insulin sensitivity being associated with muscle capillarization in older adults. ${ }^{23,41}$ In the present study, there was a correlation between average fibre capillary contacts and previously determined mixed meal myofibrillar protein synthetic response, ${ }^{10}$ which highlights the importance of an adequate microcirculation and nutrient delivery to support muscle mass. However, capillaries are also an important contributor to the SC niche ${ }^{18}$ and can influence their post-exercise activation. ${ }^{19}$ Moreover, a greater number of capillary contacts per fibre are associated with greater training-induced fibre hypertrophy in older muscle. ${ }^{20}$ In as much as there was a contraction in both the SC pool and capillary density in the present study, these inactivityinduced changes may have been mediated in part through apoptosis given the potentially greater susceptibility of SC and endothelial cells to regulated cell death with age. ${ }^{46}$ Thus, our results support the use of LLRE to maintain or enhance skeletal muscle morphology during periods of inactivity in older adults. 
In addition to maintaining muscle mass and anabolic sensitivity, ${ }^{10}$ we demonstrate that LLRE is associated with greater muscle fibre area, SC content, and capillarization in older men after 2 weeks of inactivity. Importantly, the observed differences in muscle morphology occurred in response to only six sessions of LLRE, which is supportive of exercise countermeasures to prevent and/or recover from inactivity/disuse in older populations. ${ }^{12}$ The congruence between muscle fibre size, SC content, and estimates of basal myofibrillar protein synthesis in the present study provides further evidence of a coordinated response between muscle protein metabolism and muscle stem cell activity, both of which have been independently shown to be an important regulator of muscle mass.

\section{Acknowledgements}

D. R. M. was supported in part by a Natural Sciences and Engineering Research Council (NSERC) of Canada post-doctoral fellowship, and T. A. C.-V. was supported by an NSERC postgraduate scholarship. Funding for analysis provided in part by NSERC grants to D. R. M., S. M. P., G. P., and A. P. J. The authors certify that they comply with the ethical guidelines for authorship and publishing of the Journal of Cachexia, Sarcopenia and Muscle. ${ }^{47}$

\section{Conflicts of interest}

The authors report no conflict of interest.

\section{References}

1. Cruz-Jentoft AJ, Baeyens JP, Bauer JM, Boirie $Y$, Cederholm $T$, Landi $F$, et al. Sarcopenia: European consensus on definition and diagnosis: report of the European Working Group on Sarcopenia in Older People. Age Ageing 2010;39:412-423.

2. Moore DR. Keeping older muscle "young" through dietary protein and physical activity. Adv Nutr 2014;5:599s-607s.

3. English KL, Paddon-Jones D. Protecting muscle mass and function in older adults during bed rest. Curr Opin Clin Nutr Metab Care 2010;13:34-39.

4. Dirks ML, Wall BT, Nilwik R, Weerts $D H$, Verdijk LB, van Loon L. Skeletal muscle disuse atrophy is not attenuated by dietary protein supplementation in healthy older men. J Nutr 2014;144:1196-1203.

5. Pisot R, Marusic U, Biolo G, Mazzucco $S$, Lazzer S, Grassi B, et al. Greater loss in muscle mass and function but smaller metabolic alterations in older compared with younger men following 2 wk of bed rest and recovery. J Appl Physiol (1985) 2016;120:922-929.

6. Hvid L, Aagaard P, Justesen L, Bayer ML, Andersen JL, Ortenblad N, et al. Effects of aging on muscle mechanical function and muscle fiber morphology during short-term immobilization and subsequent retraining. J Appl Physiol (1985) 2010;109:1628-1634.

7. Suetta C, Hvid LG, Justesen L, Christensen U, Neergaard K, Simonsen L, et al. Effects of aging on human skeletal muscle after immobilization and retraining. J Appl Physiol (1985) 2009;107:1172-1180.

8. Bell KE, von Allmen MT, Devries MC, Phillips SM. Muscle disuse as a pivotal problem in sarcopenia-related muscle loss and dysfunction. J Frailty Aging 2016;5:33-41.

9. Breen L, Stokes KA, Churchward-Venne TA, Moore DR, Baker SK, Smith K, et al. Two weeks of reduced activity decreases leg lean mass and induces "anabolic resistance" of myofibrillar protein synthesis in healthy elderly. I Clin Endocrinol Metab 2013;98:2604-2612.

10. Devries $M C$, Breen $L$, Von Allmen $M$ MacDonald MJ, Moore DR, Offord EA et al. Low-load resistance training during step-reduction attenuates declines in muscle mass and strength and enhances anabolic sensitivity in older men. Phys Rep 2015;3:e12493.

11. Burd NA, West DW, Staples AW, Atherton PJ, Baker JM, Moore DR, et al. Low-load high volume resistance exercise stimulates muscle protein synthesis more than highload low volume resistance exercise in young men. PLoS One 2010;5:e12033.

12. Phillips SM, Winett RA. Uncomplicated resistance training and health-related outcomes: evidence for a public health mandate. Curr Sports Med Rep 2010;9:208-213.

13. Snijders T, Parise G. Role of muscle stem cells in sarcopenia. Curr Opin Clin Nutr Metab Care 2017;20:186-190.

14. McKay BR, Ogborn DI, Bellamy LM, Tarnopolsky MA, Parise G. Myostatin is associated with age-related human muscle stem cell dysfunction. FASEB journal : official publication of the Federation of American Societies for Experimental Biology 2012;26:2509-2521.

15. Snijders T, Verdijk LB, Smeets JS, McKay BR, Senden JM, Hartgens F, et al. The skeletal muscle satellite cell response to a single bout of resistance-type exercise is delayed with aging in men. Age (Dordr) 2014;36:9699.

16. Nilwik R, Snijders $T$, Leenders M, Groen BB, van Kranenburg J, Verdijk LB, et al. The decline in skeletal muscle mass with aging is mainly attributed to a reduction in type II muscle fiber size. Exp Gerontol 2013;48:492-498.
17. Lexell J, Taylor CC, Sjostrom M. What is the cause of the ageing atrophy? Total number, size and proportion of different fiber types studied in whole vastus lateralis muscle from 15- to 83-year-old men. J Neurol Sci 1988;84:275-294.

18. Abou-Khalil R, Mounier R, Chazaud B. Regulation of myogenic stem cell behavior by vessel cells: the "menage a trois" of satellite cells, periendothelial cells and endothelial cells. Cell Cycle 2010;9:892-896.

19. Nederveen JP, Joanisse $S$, Snijders $T$, Ivankovic V, Baker SK, Phillips SM, et al. Skeletal muscle satellite cells are located at a closer proximity to capillaries in healthy young compared with older men. J Cachexia Sarcopenia Muscle 2016;7:547-554.

20. Snijders $T$, Nederveen JP, Joanisse $S$, Leenders $M$, Verdijk LB, van Loon $L$, et al. Muscle fibre capillarization is a critical factor in muscle fibre hypertrophy during resistance exercise training in older men. J Cachexia Sarcopenia Muscle 2017;8:267-276.

21. Frontera WR, Hughes VA, Fielding RA, Fiatarone MA, Evans WJ, Roubenoff R. Aging of skeletal muscle: a 12-yr longitudinal study. J Appl Physiol (1985) 2000;88:1321-1326.

22. Nederveen JP, Snijders T, Joanisse S, Wavell CG, Mitchell CJ, Johnston LM, et al. Altered muscle satellite cell activation following 16 wk of resistance training in young men. Am J Physiol Regul Integr Comp Physiol 2017;312:R85-R92.

23. Snijders $T$, Nederveen JP, Verdijk LB, Houben A, Goossens GH, Parise G, et al. Muscle fiber capillarization as determining factor on indices of insulin sensitivity in humans. Phys Rep 2017;5:e13278.

24. Churchward-Venne TA, Cotie LM, MacDonald MJ, Mitchell CJ, Prior T, Baker SK, et al. Citrulline does not enhance blood flow, microvascular circulation, or myofibrillar 
protein synthesis in elderly men at rest or following exercise. Am J Physiol Endocrinol Metab 2014;307:E71-E83.

25. Winett RA, Davy BM, Marinik E, Savla J, Winett SG, Phillips SM, et al. Developing a new treatment paradigm for disease prevention and healthy aging. Transl Behav Med 2014;4:117-123.

26. Harris BA. The influence of endurance and resistance exercise on muscle capillarization in the elderly: a review. Acta Physiol Scand 2005;185:89-97.

27. Patel HP, White MC, Westbury L, Syddall HE, Stephens PJ, Clough GF, et al. Skeletal muscle morphology in sarcopenia defined using the EWGSOP criteria: findings from the Hertfordshire Sarcopenia Study (HSS). BMC Geriatr 2015;15:171.

28. Bellamy LM, Johnston AP, De Lisio M, Parise G. Skeletal muscle-endothelial cell cross talk through angiotensin II. Am J Physiol Cell Physiol 2010;299:C1402-C1408.

29. Hepple RT, Mackinnon SL, Thomas SG, Goodman JM, Plyley MJ. Quantitating the capillary supply and the response to resistance training in older men. Pflugers Arch - Eur J Physiol 1997;433:238-244.

30. Phillips SM, Glover El, Rennie MJ. Alterations of protein turnover underlying disuse atrophy in human skeletal muscle. Appl Physiol (1985) 2009;107:645-654.

31. Wall BT, Dirks ML, van Loon LJ. Skeletal muscle atrophy during short-term disuse: implications for age-related sarcopenia. Ageing Res Rev 2013;12:898-906.

32. Reidy PT, McKenzie Al, Brunker P, Nelson DS, Barrows KM, Supiano M, et al. Neuromuscular electrical stimulation combined with protein ingestion preserves thigh muscle mass but not muscle function in healthy older adults during 5 days of bed rest. Rejuvenation Res 2017;20:449-461.

33. Verdijk LB, Dirks ML, Snijders T, Prompers JJ, Beelen M, Jonkers RA, et al. Reduced satellite cell numbers with spinal cord injury and aging in humans. Med Sci Sports Exerc 2012;44:2322-2330.

34. Verdijk LB, Snijders T, Drost M, Delhaas T, Kadi F, van Loon $L$. Satellite cells in human skeletal muscle; from birth to old age. Age (Dordr) 2014;36:545-547.

35. Snijders T, Nederveen JP, Parise G. Are satellite cells lost during short-term disuseinduced muscle fiber atrophy? J App Physiol 1985;120. United States2016:1490.

36. Arentson-Lantz EJ, Paddon-Jones D, Fry CS The intersection of disuse-induced muscle atrophy and satellite cell content: reply to Snijders, Nederveen, and Parise. J App Physiol 1985;120. United States2016:1491.

37. Fulle S, Sancilio S, Mancinelli R, Gatta V, D Pietro R. Dual role of the caspase enzymes in satellite cells from aged and young subjects. Cell Death Dis 2013;4:e955.

38. Verdijk LB, Snijders T, Beelen M, Savelberg $\mathrm{HH}$, Meijer K, Kuipers $\mathrm{H}$, et al. Characteristics of muscle fiber type are predictive of skeletal muscle mass and strength in elderly men. J Am Geriatr Soc 2010;58:2069-2075.

39. Brooks NE, Myburgh KH. Skeletal muscle wasting with disuse atrophy is multidimensional: the response and interaction of myonuclei, satellite cells and signaling pathways. Front Physiol 2014;5:99.

40. Rennie MJ, Wackerhage $H$, Spangenburg $\mathrm{EE}$, Booth FW. Control of the size of the human muscle mass. Annu Rev Physiol 2004;66:799-828.
41. Hedman A, Berglund L, Essen-Gustavsson $B$, Reneland $\mathrm{R}$, Lithell $\mathrm{H}$. Relationships between muscle morphology and insulin sensitivity are improved after adjustment for intra-individual variability in 70 year-old men. Acta Physiol Scand 2000;169:125-132.

42. Timmerman KL, Lee JL, Fujita S, Dhanani S, Dreyer HC, Fry CS, et al. Pharmacological vasodilation improves insulin-stimulated muscle protein anabolism but not glucose utilization in older adults. Diabetes 2010;59:2764-2771.

43. Edgerton VR, Zhou MY, Ohira Y, Klitgaard $H$, Jiang $B$, Bell $G$, et al. Human fiber size and enzymatic properties after 5 and 11 days of spaceflight. J Appl Physiol (1985) 1995;78:1733-1739.

44. Ahmed SK, Egginton S, Jakeman PM, Mannion AF, Ross HF. Is human skeletal muscle capillary supply modelled according to fibre size or fibre type? Exp Physiol 1997;82:231-234.

45. Barnouin $Y$, McPhee JS, Butler-Browne G, Bosutti A, De Vito G, Jones DA, et al. Coupling between skeletal muscle fiber size and capillarization is maintained during healthy aging. J Cachexia Sarcopenia Muscle 2017;8:647-659.

46. Wang $\mathrm{H}$, Listrat $A$, Meunier B, Gueugneau M, Coudy-Gandilhon C, Combaret L, et al. Apoptosis in capillary endothelial cells in ageing skeletal muscle. Aging Cell 2014;13:254-262.

47. von Haehling S, Morley JE, Coats AJS, Anker SD. Ethical guidelines for publishing in the Journal of Cachexia, Sarcopenia and Muscle: update 2017. J Cachexia Sarcopenia Muscle 2017;8:1081-1083. 